\title{
Decomposition of abnormal free locomotor behavior in a rat model of Parkinson's disease
}

\author{
Benjamin Grieb ${ }^{1,2 *}$, Constantin von Nicolai i,3, Gerhard Engler ${ }^{1}$, Andrew Sharott ${ }^{1,4}$, \\ Ismini Papageorgiou ${ }^{5}$, Wolfgang Hamel ${ }^{6}$, Andreas K. Engel ${ }^{1+}$ and Christian K. Moll ${ }^{1+}$ \\ ${ }^{1}$ Department of Neurophysiology and Pathophysiology, University Medical Center Hamburg-Eppendorf, University of Hamburg, Hamburg, Germany \\ 2 Department of General Psychiatry, Center for Psychosocial Medicine, University of Heidelberg, Heidelberg, Germany \\ ${ }^{3}$ Centre for Integrative Neuroscience, University of Tübingen, Tübingen, Germany \\ ${ }^{4}$ Medical Research Council, Anatomical Neuropharacology Unit, Department of Pharmacology, University of Oxford, Oxford, UK \\ ${ }^{5}$ Division of General Neurophysiology, Institute of Physiology and Pathophysiology, University of Heidelberg, Heidelberg, Germany \\ ${ }^{6}$ Department of Neurosurgery, University Medical Center Hamburg-Eppendorf, University of Hamburg, Hamburg, Germany
}

\section{Edited by:}

Hagai Bergman, The Hebrew

University, Israel

Reviewed by:

M. Gustavo Murer, Universidad de

Buenos Aires, Argentina

Nicola B. Mercuri, University of

Rome, Italy

\section{*Correspondence.}

Benjamin Grieb, Department of General Psychiatry, Center for

Psychosocial Medicine, University of

Heidelberg, Voßstrasse 2, 69115

Heidelberg, Germany

e-mail: benjamin.grieb@

med.uni-heidelberg.de

${ }^{\dagger}$ These authors have contributed

equally to this work.
Poverty of spontaneous movement, slowed execution and reduced amplitudes of movement (akinesia, brady- and hypokinesia) are cardinal motor manifestations of Parkinson's disease that can be modeled in experimental animals by brain lesions affecting midbrain dopaminergic neurons. Most behavioral investigations in experimental parkinsonism have employed short-term observation windows to assess motor impairments. We postulated that an analysis of longer-term free exploratory behavior could provide further insights into the complex fine structure of altered locomotor activity in parkinsonian animals. To this end, we video-monitored 23 hours of free locomotor behavior and extracted several behavioral measures before and after the expression of a severe parkinsonian phenotype following bilateral 6-hydroxydopamine (6-OHDA) lesions of the rat dopaminergic substantia nigra. Unbiased stereological cell counting verified the degree of midbrain tyrosine hydroxylase positive cell loss in the substantia nigra and ventral tegmental area. In line with previous reports, overall covered distance and maximal motion speed of lesioned animals were found to be significantly reduced compared to controls. Before lesion surgery, exploratory rat behavior exhibited a bimodal distribution of maximal speed values obtained for single movement episodes, corresponding to a "first" and "second gear" of motion. 6-OHDA injections significantly reduced the incidence of second gear motion episodes and also resulted in an abnormal prolongation of these fast motion events. Likewise, the spatial spread of such episodes was increased in 6-OHDA rats. The increase in curvature of motion tracks was increased in both lesioned and control animals. We conclude that the discrimination of distinct modes of motion by statistical decomposition of longer-term spontaneous locomotion provides useful insights into the fine structure of fluctuating motor functions in a rat analog of Parkinson's disease.

Keywords: 6-OHDA lesions, stereology, spontaneous activity, Parkinson disease, video monitoring

\section{INTRODUCTION}

Neurotoxin-induced degeneration of nigral dopamine neurons in experimental animals results in motor abnormalities relevant to motor symptoms of Parkinson's disease (PD; Cenci et al., 2002). One strategy to deplete midbrain dopaminergic neurons in rats is to infuse the neurotoxin 6-hydroxydopamine (6-OHDA) directly into the substantia nigra pars compacta ( $\mathrm{SNc}$; Schwarting and Huston, 1996a,b), which can also cause moderate cell death in the neighboring ventral tegmental area (VTA). In the prototypic toxin-induced rat model of PD, unilateral intracerebral 6-OHDA injections lead to the expression of a strictly lateralized hemiparkinsonian phenotype, the behavioral sequelae of which have been described in great detail. A wide variety of different behavioral tests are used to examine motor changes associated with the asymmetrical depletion of the nigrostriatal dopaminergic system, e.g., skilled motor tasks (Mokrý et al., 1995; Truong et al., 2006), footprint-analysis (Metz et al., 2005), treadmill running (Brazhnik et al., 2012), or drug-induced rotation (Ungerstedt, 1971; Kelly, 1975). Compared to the unilateral 6-OHDA model, bilaterally lesioned rats have been used less commonly, although they display a far more severe parkinsonian phenotype. In this respect, the slowness and scarcity of movement observed in the bilateral 6-OHDA rat model resembles more closely the marked expression of PD-like symptoms in monkeys treated systemically with 1-methyl-4-phenyl-1,2,3,6tetrahydropyridine (Bergman et al., 1990) and cardinal motor manifestations of human PD patients in advanced disease stages. The development of pronounced motor impairment in rats with severe bilateral 6-OHDA lesions is, however, often accompanied by aphagia, adipsia and abulia (Schallert et al., 1978; Sakai and Gash, 1994; Cass et al., 2005; Ferro et al., 2005).

Rat equivalents of akinesia are commonly assessed in an "open-field" environment. Most often, rat motion (including scanning, walking or running, as well as rearing) is detected by 
consecutively breaking light beams arranged in an array around the arena (Cass et al., 2005; Ferro et al., 2005; Belujon et al., 2007). Other methods to quantify locomotor capacities are wheel (Schallert et al., 1978) or treadmill running (Avila et al., 2010). These standard measurements usually assess a short period of 10-60 min of locomotor activity (Cass et al., 2005; Ferro et al., 2005; Belujon et al., 2007), assuming a stable motor phenotype over time. However, these methods may underestimate the complexity of exploratory behavior, in particular long-term motor fluctuations.

Recently, a novel analytical approach to video-based tracking data utilized the statistical discrimination of rat motor behavior on the basis of speeds (Drai et al., 2000; Drai and Golani, 2001). This form of data analysis showed that naïve rats, but also naïve mice (Drai et al., 2001), use distinctly different modes of motion to explore their environment. The current study aimed to assess the impact of dopamine depletion on these naturally occurring behavioral patterns. We hypothesized that expression of a severe parkinsonian phenotype would not only reduce overall motion speed, as could be assessed with other standard quantification methods, but would also alter the fine spatio-temporal structure of exploratory locomotion modes. To test this, we adapted the analysis approach of Drai and colleagues to video-based tracking data from 23 hours of continuous and spontaneous locomotion of bilaterally 6-OHDA lesioned and control rats.

\section{MATERIALS AND METHODS ANIMALS}

Animal experiments were approved by the local government authorities of Hamburg and carried out in accordance with the European Council Directive 86/609/EEC. All experiments were performed on male Brown Norway rats (Rattus norvegicus; Charles River Laboratories, Sulzfeld, Germany). All efforts were made to minimize suffering. The bilateral 6-OHDA lesion model is known to be associated with high mortality due to aphagia/adipsia and following loss of body weight (Ferro et al., 2005). Careful clinical inspection and weighting of animals was performed on a daily basis. In general, we offered a soft and moist rat chow and 10\%-glucose solution in addition to standard chow and water ad libitum. To sustain aphagic and adipsic PD rats, we administered a liquid and high caloric nutrition for rodents (Altromin, Lage, Germany) by manual needle feeding. In total, the dropout rate was 5/14 PD rats. Dropouts included perioperative death $(n=2 \mathrm{PD})$ and loss of $>20 \%$ body weight without stabilization of weight loss within 14 days after surgery ( $n=3 \mathrm{PD}$ ) which led to euthanasia of these animals by decapitation under deep anesthesia (i.p.-injection of ketamine $100 \mathrm{mg} / \mathrm{kg}$, xylazine $6 \mathrm{mg} / \mathrm{kg}$ ).

\section{EXPERIMENTAL DESIGN}

We randomly assigned 14 rats to a $\mathrm{PD}$ group receiving bilateral 6-OHDA injections into the SNc and eight rats to a control group receiving bilateral vehicle injections (preoperative weight: $384 \pm 32 \mathrm{~g}$, mean $\pm S D$ ). Video tracking of 23 hours of spontaneous locomotor activity was performed prior to surgery. Due to the labor intensity of sustaining PD rats we ran the experiment in two phases. Within the first subset of 10 rats $(n=8 \mathrm{PD} ; n=2$ controls) the dropout rate was $4 / 8 \mathrm{PD}$ rats. The second subset of 12 rats $(n=6 \mathrm{PD} ; n=6$ controls) exhibited a dropout rate of $1 / 6$ PD rats. Postoperative locomotor activity was assessed after a recovery period of $12 \pm 2$ days in the first subset and $28 \pm 2$ days in the second subset. After postoperative video monitoring, all rats were subsequently used in a separate study. We sacrificed rats after induction of deep anesthesia (ketamine/xylazine) $\sim 14$ weeks after lesioning by transcardial perfusion with saline. A midbrain tissue block was immersion-fixed in 4\%-PFA-solution (paraformaldehyde in $0.1 \mathrm{M}$ phosphate buffered saline, SigmaAldrich) for subsequent histological $\mathrm{TH}$-staining and stereological counting of tyrosine hydroxylase $(\mathrm{TH})$-positive $\mathrm{SNc}$ and VTA neurons.

\section{BILATERAL 6-HYDROXYDOPAMINE LESIONS}

Stereotactic injections were performed under general anesthesia introduced with isoflurane (Baxter Germany $\mathrm{GmbH}$, Unterschleißheim, Germany) and maintained with i.p.-injections of ketamine ( $65 \mathrm{mg} / \mathrm{kg}$, Dr. E. Gräub AG, Bern, Switzerland) and xylazine $(3 \mathrm{mg} / \mathrm{kg}$, Bayer Health Care, Leverkusen, Germany). Thirty minutes prior to 6-OHDA or vehicle injections, rats received a bolus i.p.-injection of desipramine $(25 \mathrm{mg} / \mathrm{kg}$, SigmaAldrich, Munich, Germany) to minimize uptake of 6-OHDA in noradrenergic midbrain neurons (Schwarting and Huston, 1996b). Cardiopulmonary protection was assured by an initial bolus injection of atropine $(0.25 \mathrm{mg} / \mathrm{kg}, \mathrm{B}$. Braun Melsungen AG, Melsungen, Germany). Body temperature was monitored during surgery with a rectal probe and hypothermia was prevented with an adjustable heating pad (FST, Heidelberg, Germany). In addition, the eyes were covered with dexpanthenol cream to prevent exsiccation. Animals were mounted in a stereotactic frame (David Kopf Instruments, Tujunga, USA). To target the SNc a Hamilton microliter-syringe (FST) was lowered through a burr hole placed at $+4 \mathrm{~mm}$ AP and $\pm 2.2 \mathrm{~mm}$ ML using the interaural line as reference (Paxinos and Watson, 2005). The syringe was slowly lowered to the target depth at $-8 \mathrm{~mm}$ relative to the dura. Five microliter neurotoxin ( $3 \mu \mathrm{g} / \mu \mathrm{l}$ 6-OHDA hydrochloride free base in $0.2 \%$ ascorbic acid solution, stored on ice; Sigma-Aldrich) or vehicle (aqua injectabilia, $0.2 \%$ ascorbic acid solution; Sigma-Aldrich) was slowly infused at a rate of $0.5 \mu \mathrm{l} / \mathrm{min}$ and the syringe was left in place for $2 \mathrm{~min}$ to allow for complete absorption of the toxin. The burr hole was closed with bone wax and the procedure was repeated in the contralateral hemisphere. Rats received s.c.-injections of metamizole $(100 \mathrm{mg} / \mathrm{kg}$, Medistar, Holzwickede, Germany) for postoperative analgesia following surgery and on behavioral signs of pain distress.

\section{STEREOLOGY}

To count TH-positive dopaminergic cells in PD rats and controls we performed free-floating TH-immunohistochemistry on serial $40 \mu \mathrm{m}$ coronal sections of the midbrain. Figure 1A displays representative examples of photomicrographs depicting $\mathrm{TH}$-stained midbrain sections from a vehicle and 6-OHDA injected rat. Due to technical reasons we could only obtain stereology in a subset of 8/9 PD-rats and 5/8 controls. PFA-fixed tissue blocks containing SNc and VTA were transferred to 30\%-sucrose solution and kept at $4^{\circ} \mathrm{C}$ for 24 hours. Sections were cut with a freezing-microtome 

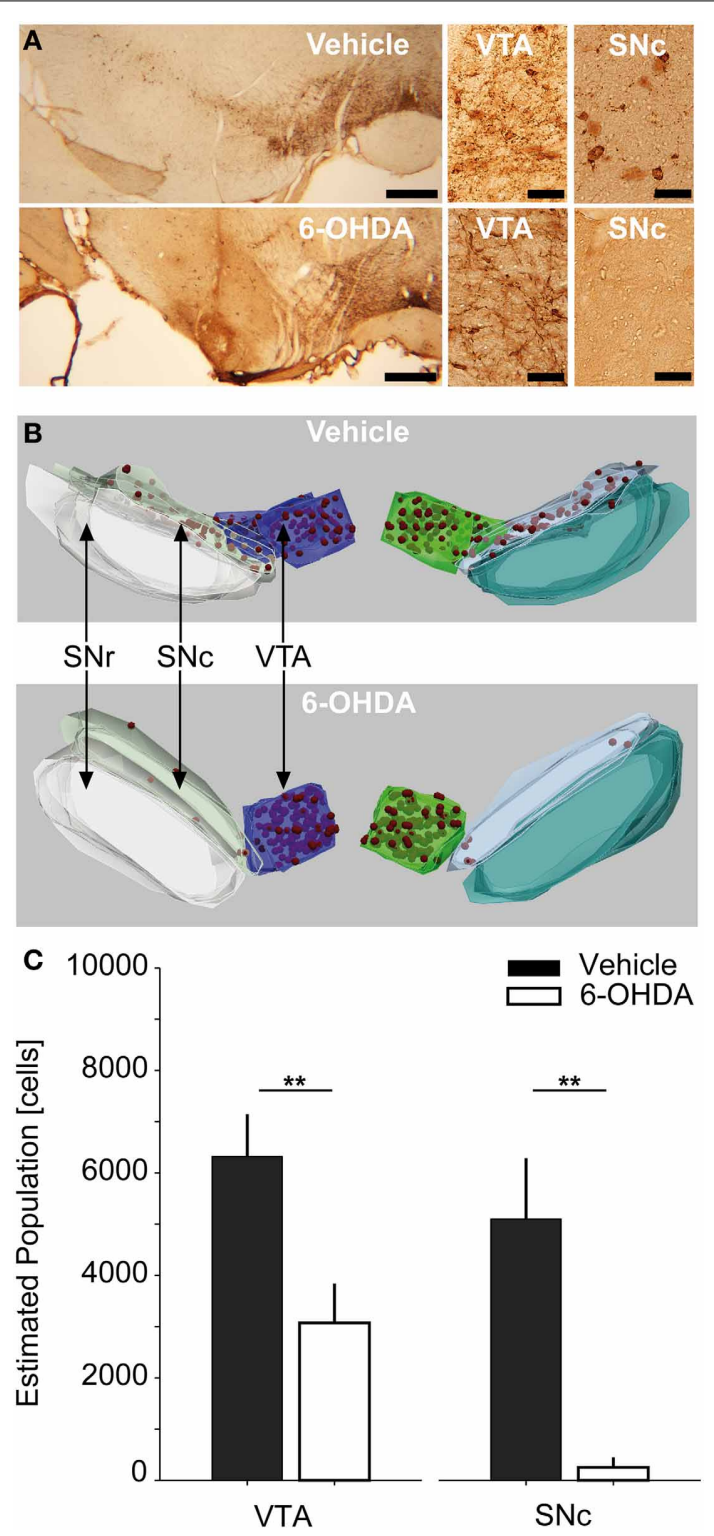

FIGURE 1 | Stereological counting of TH-positive SNc and VTA neurons. (A) Representative photomicrographs of $\mathrm{TH}$-stained midbrain sections of a vehicle and 6-OHDA injected rat (left column, scale bar equals $500 \mu \mathrm{m}$ ) as well as $40 \times$ magnifications of VTA and SNc-ROls (middle and right column, scale bar equals $50 \mu \mathrm{m}$ ). (B) 3D-reconstructions of stereological regions of interest for the same vehicle and 6-OHDA injected rats as in (A). Red spheres indicate stereologically counted cells in the SNc and VTA. (C) Estimated absolute population of cells in the SNc and VTA for vehicle $(n=5)$ and 6-OHDA $(n=8)$ injected rats. Double asterisks denote $P \leq 0.001$ (uncorrected). TH, tyrosine-hydroxylase; 6-OHDA,

6-hydroxydopamine; VTA, ventral tegmental area; SNc, substantia nigra pars compacta; SNr, substantia nigra pars reticulata.

(Leica Instruments, Wetzlar, Germany) and stained in freefloating fashion for TH-activity. Briefly, sections were washed in phosphate buffer (0.01 M PBS, Sigma-Aldrich), incubated with $3 \%-\mathrm{H}_{2} \mathrm{O}_{2}$-solution for $3 \mathrm{~min}$ to block endogenous peroxidase activity and incubated with $2 \%$ normal horse serum (added with
$0.3 \%$ Triton X-100, Sigma-Aldrich) for $30 \mathrm{~min}$. Sections were then incubated over night at $4{ }^{\circ} \mathrm{C}$ with the primary $\mathrm{TH}$-antibody (1:250, monoclonal mouse antibody, Novocastra reagents, Leica Microsystems, Wetzlar, Germany), followed by the biotinylated secondary antibody (1:400, Novocastra reagents) for $30 \mathrm{~min}$, and afterwards incubated with avidin and biotinylated horseradish peroxidase (ABC kit, Novocastra reagents) for another $30 \mathrm{~min}$. $\mathrm{TH}$ was visualized by adding peroxidase substrate $(0.02 \%$ DAB reagent in $0.003 \% \mathrm{H}_{2} \mathrm{O}_{2}$ in PBS) for 2-10 min duration. Finally, sections were mounted on glass slides, dehydrated in an increasing alcohol row and fixed under a cover slid with Roti Histokitt II (Carl Roth, Karlsruhe, Germany).

Unbiased stereological counting of TH-positive cells in the SNc and VTA was performed using the Stereoinvestigator Software (Version 10.0, MicroBrightField Inc., Williston, Vermont, USA). The hardware consisted of an Olympus Bx61 brightfield microscope (Olympus Deutschland $\mathrm{GmbH}$, Hamburg, Germany) equipped with a Microfire TM A/R camera (Optronics, California, USA) and an $\mathrm{x}-\mathrm{y}-\mathrm{z}$ galvano table (Carl Zeiss AG, Jena, Germany). The optical fractionator probe (West et al., 1991; West, 2002) was applied on series of $40 \mu \mathrm{m}$ thick coronal sections. To assure sampling of comparable structural parts, the stereological analysis was centered on an independent anatomical hallmark (the rootlets of the oculomotor cranial nerve). Overall we sampled 10 sections (sampling rate of $1.2 \pm 0.3$ mean $\pm S D$ sections) spanning $\sim 480 \mu \mathrm{m}$ in the cranio-caudal dimension. However, due to availability of continuous $\mathrm{TH}$-sections containing $\mathrm{SNc}$ and VTA, three rats were investigated using 7,8 , or 9 sections, respectively. Using a $2.5 \times$ magnification with numerical aperture of 0.075 we defined three anatomical regions of interest (ROI), i.e., the $\mathrm{SNc}$, the VTA and the substantia nigra pars reticulata $(\mathrm{SNr}$ ) (Paxinos and Watson, 2005). For examples of anatomical 3-D reconstructions of ROIs and counted cells, see Figure 1B. Cell counting was performed using a $40 \times$ Plan-Neofluar dry type objective lens with a numerical aperture of 0.75 (Carl Zeiss AG) within the SNc and VTA-ROIs. The counting frame $(50 \times 50 \mu \mathrm{m})$ with dissector height of $20 \mu \mathrm{m}$ was applied with a uniform random sampling grid of $150 \times 150 \mu \mathrm{m}$ (optical dissector volume of $50,000 \mu \mathrm{m}^{3}$, sampling grid area of 22,500 $\mu \mathrm{m}^{2}$, Gundersen, 1986). Schaeffer's estimated coefficient of error ranged between 0.05 and 0.12 for the VTA of both controls and PD animals, as well as the SNc of controls. For SNc-ROIs of PD animals it ranged between 0.3 and 0.9 , reflecting the scarceness of $\mathrm{TH}$-positive cells in this area (Gundersen, 1986; West, 2002). The total volume of the sampled SNc and VTA parts was stereologically estimated using the Cavalieri method (Gundersen and Jensen, 1987). Our histological regime resulted in robust sampling $>200$ neurons per VTA and SNc in vehicle injected controls, enabling us to express $\mathrm{TH}$-positive cell numbers in absolute numbers. Cell counts were calculated separately for each ROI and hemisphere, respectively, and combined across hemispheres $(n=6$ hemispheres per group).

To assess the symmetry of depletion we calculated the laterality index $(L I)$ for each rat (Seghier, 2008), $L I=f \times\left(E_{L H}-\right.$ $\left.E_{R H}\right) /\left(E_{L H}+E_{R H}\right)$, where $E_{L H}$ and $E_{R H}$ are the combined VTA and $\mathrm{SNc}$ estimates of $\mathrm{TH}$-positive cells for the left and right 
hemisphere, respectively, and $f$ is a scaling factor set to 1 resulting in the $L I$ to be bound between -1 and 1 .

\section{BEHAVIORAL MONITORING}

Locomotion of 6-OHDA or vehicle injected rats was investigated via continuous video monitoring of 23 hours of spontaneous behavior in an open field-like environment. We used an infrared video-based tracking system (VideoMot 2.0, TSE Systems, Bad Homburg, Germany) to record movement paths as time series of $\mathrm{x}$-y-positions at a sampling rate of $12.8 \mathrm{~Hz}$. The recording arena was spaced $70 \times 100 \mathrm{~cm}$ with $40 \mathrm{~cm}$ high walls (i.e., $320 \times 435$ pixel after frame grabbing and offline analysis) and equipped with three food pellet feeders and one water outlet positioned in the corners. The ground was covered with bedding. The arena size was chosen to be $\sim 3$ times larger than the size of the rat's home cage to allow for generation of more naturalistic movement patterns including running. The arena was placed in a custommade recording box lined with foamed plastic to ensure light and acoustic insulation. Recordings took place under constant darkness.

\section{BEHAVIORAL DATA ANALYSIS}

To analyze spontaneous long-term behavior we modified a data processing and analysis approach developed for open-field locomotion in rodents (Drai et al., 2000; Drai and Golani, 2001). All routines were written in MATLAB (The Mathworks, Natick, USA). The analytical approach is based on the idea of calculating single speed values $(\mathrm{cm} / \mathrm{s})$ from $x-y$-position time series using a sliding window, separating rest vs. motion episodes and characterizing motion episodes by the maximal speed reached within each episode rather than the average speed of a given episode. Our modified analysis consisted of five separate analysis steps (see Figure 2): First, raw x-y-position time series data obtained for 23 hours at a sampling rate of $12.8 \mathrm{~Hz}(\sim 1,000,000$ single data points) contained large amounts of artifacts (see Figure $2 \mathbf{A}$ ). Typically, artifacts resulted from spurious "jumping" within a continuous $\mathrm{x}-\mathrm{y}$-position time series to distant places and back. Frequent sources of artifacts lay outside the actual recording arena and could therefore be removed through spatial outlier rejection. However, artifacts also appeared within the arena, generally jumping toward the arena's corners where animals spent most of their resting time (Figure 2B). All places on the pixel level that were "visited" twice during the recording time were neglected, eliminating artifacts associated with resting spots (Figure 2C). To further de-noise the recordings we applied a low-pass threshold of $80 \mathrm{~cm} / \mathrm{s}$ (Figure 2D), as preliminary data screening showed that no rat reached speed levels $>70 \mathrm{~cm} / \mathrm{s}$ inside the recording arena (data not shown). Single speed values were calculated on position time series with a moving window of $0.3 \mathrm{~s}$ (Drai et al., 2000; Drai and Golani, 2001). Separation of resting vs. motion episodes was done by high-pass thresholding speed values at a noise level of $4 \mathrm{~cm} / \mathrm{s}$ (Figure 2E). This threshold was based on previous work of Drai et al. (2000) and adapted to the distribution of single speeds in our data set with a peak at $4 \mathrm{~cm} / \mathrm{s}$ (data not shown). The remaining motion episodes were used for further analysis (Figure 2F). To compensate for erroneous separation of movement episodes resulting from artifact correction we interpolated $\mathrm{x}-\mathrm{y}$-coordinates of single missing values.

\section{BEHAVIORAL ENDPOINTS}

Smoothed histograms of log transformed maximal speed values, termed "log max-SD," were used to identify different modes of motion in control and PD rats (Drai et al., 2000; Drai and Golani, 2001). A Gaussian mixture model was fitted toward the empirical data to obtain information about the localization of different "gears" of motion within the distribution of speeds. Gears were separated at $10 \mathrm{~cm} / \mathrm{s}$, a value consistently derived from preoperative recordings. Descriptive locomotion parameters were calculated for slow (i.e., first gear) and fast (i.e., second gear) episodes. However, as the dissection of second gear episodes allowed a view on full-blown motion, we concentrated our analysis on such episodes.
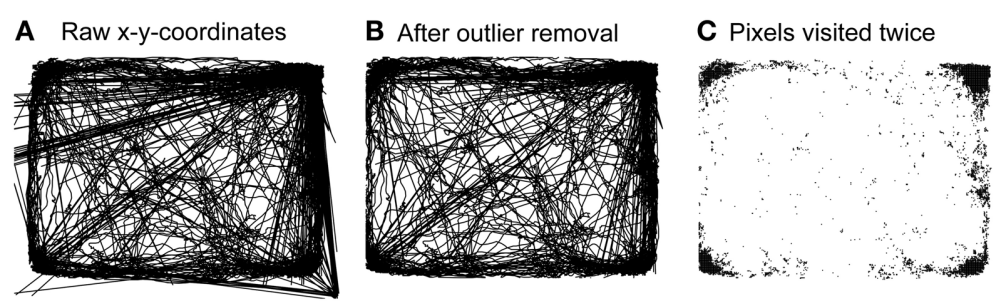

D Low-pass threshold

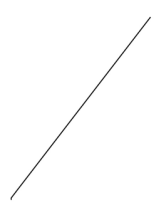

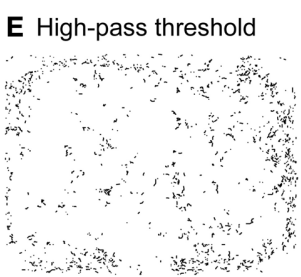

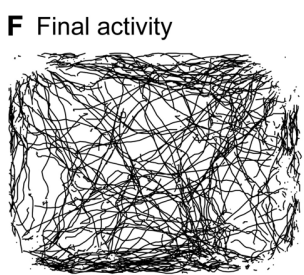

FIGURE 2 | Steps of behavioral analysis. Activity of a representative rat during 23 hours of pre-operative monitoring. (A) Raw activity including all artifacts. (B) Activity remaining after outlier rejection. (C) Spaces on the single pixel level that were "visited" twice during the whole recording time. (D) Artifacts excluded through application of a low-pass threshold of $80 \mathrm{~cm} / \mathrm{s}$. (E) Resting activity excluded through application of a high-pass threshold of $4 \mathrm{~cm} / \mathrm{s}$. (F) Activity used for subsequent behavioral analyses. 
The six different behavioral endpoints were defined as: (1) incidence of fast speed movement episodes, which is the percentage of second gear episodes relative to the number of all motion episodes; (2) absolute distance, calculated as the cumulative sum of the overall covered distance for all motion episodes; (3) average maximal movement speed, corresponding to the mean of all maximal speeds of second gear episodes; (4) dwell time, i.e., the average time it took the rats to execute a motion episode; (5) spatial spread, i.e., the mean distance covered within a motion episode; (6) curvature of movement tracks, i.e., the ratio of the detected real motion track distance and the distance of the virtual line connecting two coordinate pairs directly during temporal windows of $0.5 \mathrm{~s}$. Curvatures were averaged across all windows of a given motion episode.

Our experimental paradigm allowed for post-hoc splitting of the experimental groups into rats monitored after $12 \pm 2$ days and $28 \pm 2$ days weeks of recovery. We separately analyzed the correlation between the six behavioral endpoints and the estimated absolute population of TH-positive neurons in the SNc and VTA, the corresponding LI of cell depletion and the observed weight reduction for the 6-OHDA and vehicle injected control group. We utilized bootstrap regressions, which do not rely on a normal distribution of data.

\section{STATISTICAL ANALYSIS}

All post-hoc analyses were performed using the MATLAB Statistics Toolbox and the BRAVO Toolbox for Bootstrap Regression Analysis of Voxelwise Observations. In case of non-normal data distributions, we employed non-parametric statistical testing (Wilcoxon rank sum test). An alpha level of 0.05 was used for all statistical tests. All statistical results are given as mean \pm standard deviation (SD). We corrected $P$-values for multiple comparisons using the false discovery rate (FDR) method (Benjamini and Hochberg, 1995) in case of descriptive behavioral results and multiple bootstrap regression analyses. Bootstrap regressions were performed with 5000 iterations. For descriptive analysis of behavioral endpoints alone, $P$-values were FDR-corrected for a total of 48 different comparisons (2 experimental groups, 6 behavioral endpoints; 4 different comparisons: preoperative vs. $12+28$ days postoperative, preoperative vs. 12 days postoperative, preoperative vs. 28 days postoperative and 12 days postoperative vs. 28 days postoperative). For bootstrap regressions, $P$-values were FDR-corrected for a total of 55 regressions (the estimated neuronal population of the SNc and VTA, the LI, weight reduction and 6 behavioral endpoints).

\section{RESULTS}

\section{STEREOLOGICAL COUNTING OF TH-POSITIVE SNc AND VTA NEURONS}

Bilateral injections of $15 \mu \mathrm{g}$ 6-OHDA into the SNc resulted in extensive cell death of dopaminergic neurons in the SNc and intermediate cell death in the VTA (Figure 1C). The estimated population of TH-positive neurons for combined SNc-ROIs was reduced by $-95 \%$ in PD rats compared to controls. The absolute number of cells within combined SNc-ROIs was $253.8 \pm 196.9$ in PD vs. $5098 \pm 1189$ in control rats $(P=0.001)$. For combined VTA-ROIs, the estimated population of TH-positive neurons was reduced by $-51.4 \%$ in $\mathrm{PD}$ rats compared to controls. Here, the absolute number of cells within combined VTA-ROIs was $3073 \pm$ 766 in PD vs. $6318 \pm 828$ in control rats $(P=0.001)$. We also tested whether the cell estimates differed significantly between the two subgroups that were monitored 12 and 28 days after lesioning. No statistically significant difference was found for either VTA or SNc-ROIs of 6-OHDA or vehicle injected rats ( $P$-values $>$ 0.25 , data not shown). Furthermore, the estimated populations within single ROIs did not differ significantly between left vs. right hemispheres in $\mathrm{PD}$ and control rats, respectively $(P>0.2)$. Likewise, the LI for 6-OHDA treated rats was $0.14 \pm 0.2$ and $0.08 \pm 0.01$ for controls $(P=1$, data not shown $)$.

\section{POSTSURGICAL COURSE}

Careful daily inspection of PD and control rats in the home cage environment revealed reduced spontaneous locomotion and movement speed of 6-OHDA-treated rats. Body posture appeared with a hunchback-like shape and hind limb rigidity was detectable upon manual assessment. PD rats displayed aphagia and adipsia leading to a reduction in body weight of $19 \pm 7.7 \%$ of the preoperative weight in $\mathrm{PD}$ and $1.5 \pm 4.5 \%$ in control rats prior to postoperative behavioral monitoring $(P=0.002)$. Three PD rats were euthanized, as they did not exhibit stabilization of weight loss within 12 days after surgery. None of the control rats showed overt movement deficits, aphagia, or adipsia. No additional signs of altered behavior that could indicate persistent pain distress were detected during inspections.

\section{CIRCADIAN ACTIVITY}

Free locomotion monitoring took place under constant dark conditions for at least 23 continuous hours. Although no external light cues were given we saw a pattern of locomotor activity reflecting a circadian rhythm in preoperative (data not shown) and postlesion recordings in vehicle injected controls (Figure 3A). In most rats we found two activity phases that were separated by a phase of reduced activity lasting $\sim 12$ hours each.

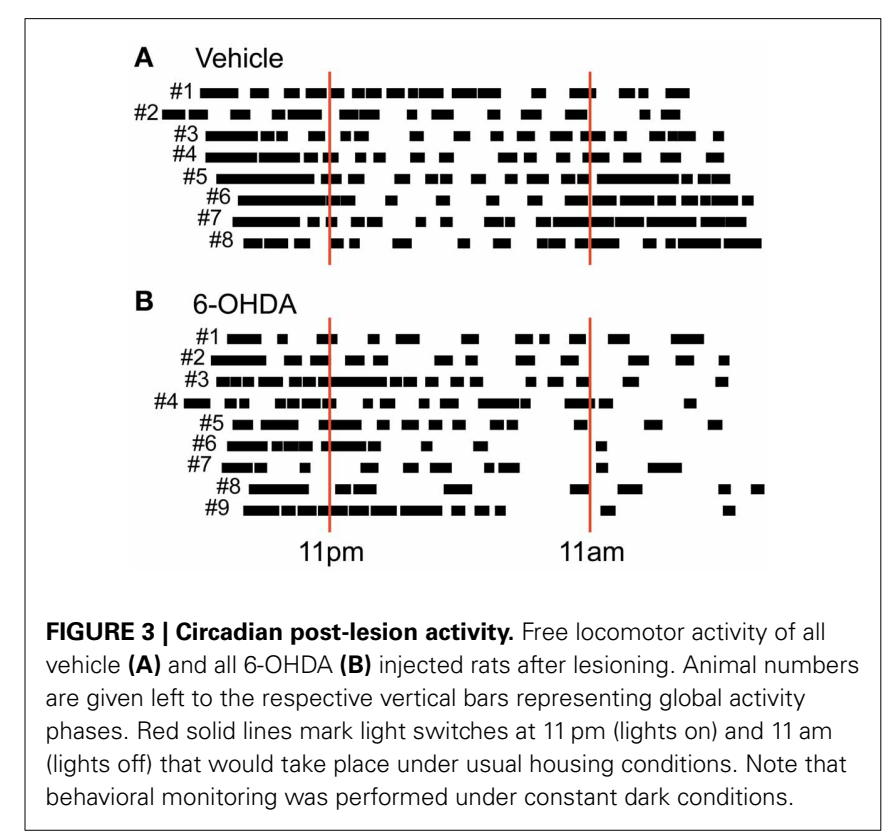


This corresponds to the fact that our rats were accustomed to a 12 hour day/night cycle switching at 11 am (lights off) and $11 \mathrm{pm}$ (lights on). Experiments were usually started between 5 and $6 \mathrm{pm}$ and held under constant dark conditions. In PD rats, however, we found a partially disturbed circadian rhythm (Figure 3B) with two major changes. First, PD rats tended to show a prolonged initial global activity period and less distinguishable transition periods. Second, PD rats did not exhibited a clear rebound of activity in the later phase of monitoring.

\section{BIMODAL DISTRIBUTION OF MAXIMAL SPEED VALUES}

In prelesion recordings, characterization of movement episodes by the log max-SD resulted in a bimodal distribution (Figure 4). In order to distinguish between distinct gears of motion, we fitted a Gaussian mixture model to the empirical distribution of each recording. First gear speeds were centered at a log max-SD of $0.72 \pm 0.02$ in control and $0.71 \pm 0.03$ in PD rats, corresponding to a velocity of $5.1 \mathrm{~cm} / \mathrm{s}$. Second gear speeds were centered at a log max-SD of $1.24 \pm 0.1$ in control and $1.24 \pm 0.06$ in PD rats, i.e., $17.4 \mathrm{~cm} / \mathrm{s}$. Notably, 6-OHDA injections resulted in marked changes of the distribution of $\log$ max-SD values. We observed a partial loss of the bimodal distribution and curve flattening at the center of preoperative second gear episodes. This complicated the fitting of Gaussians and produced spurious results in several animals, e.g., PD rats \#1, \#2, and \#5. Therefore, we separated gears at a $\log$ max-SD of $1(10 \mathrm{~cm} / \mathrm{s})$, a value derived from the consistent bimodal distributions of preoperative monitoring.

\section{INCIDENCE OF FAST SPEED MOVEMENT EPISODES}

Comparing the number of first gear and second gear episodes per recording, we saw a shift in their incidence after 6-OHDA lesioning (Figure 5A). In the PD group, the incidence of second gear episodes decreased from $50.6 \pm 4.1 \%$ to $36.4 \pm 9 \%$ (pre- vs. $12+$ 28 days postoperatively, $P=0.01$, corrected). In vehicle injected controls the proportion of first gear and second gear episodes remained the same $(51.8 \pm 6.1 \%$ vs. $47.4 \pm 4.7 \%$, pre- vs. $12+$ 28 days postoperatively, $P=0.26$, corrected). Considering the two subpopulations separately, we only saw a significant difference after 12 days of recovery in $\mathrm{PD}$ rats $(P=0.02$ at 12 days vs. $P=0.08$ at 28 days; $P=0.35$ for 12 vs. 28 days; corrected).

\section{OVERALL COVERED DISTANCE}

6-OHDA injections led to a reduction of the total covered distance within 23 hours of spontaneous locomotion (PD rats: $129.9 \pm$ $25.8 \mathrm{~m}$ vs. $94.3 \pm 28.7 \mathrm{~m}$, pre- vs. $12+28$ days postoperatively, $P=0.05$; control rats: $143.2 \pm 38 \mathrm{~m}$ vs. $138.9 \pm 31.7 \mathrm{~m}$, prevs. $12+28$ days postoperatively, $P=1$, corrected; Figure 5B). Again, this difference was significant for the PD group at 12 days, but not at 28 days of recovery $(P=0.02$ at 12 days vs. $P=0.35$ at 28 days; $P=1$ for 12 vs. 28 days; corrected).

\section{AVERAGE MAXIMAL MOVEMENT SPEED}

The log max-SD across all second gear episodes of full motion was significantly reduced in PD rats $(19.9 \pm 1.7 \mathrm{~cm} / \mathrm{s}$ vs. $17 \pm$ $2 \mathrm{~cm} / \mathrm{s}$, pre- vs. $12+28$ days postoperatively, $P=0.02$; control rats: $20.6 \pm 1.8 \mathrm{~cm} / \mathrm{s}$ vs. $20 \pm 1.3 \mathrm{~cm} / \mathrm{s}$, pre- vs. $12+28$ days postoperatively, $P=1$, corrected; Figure 5C). Likewise, the difference

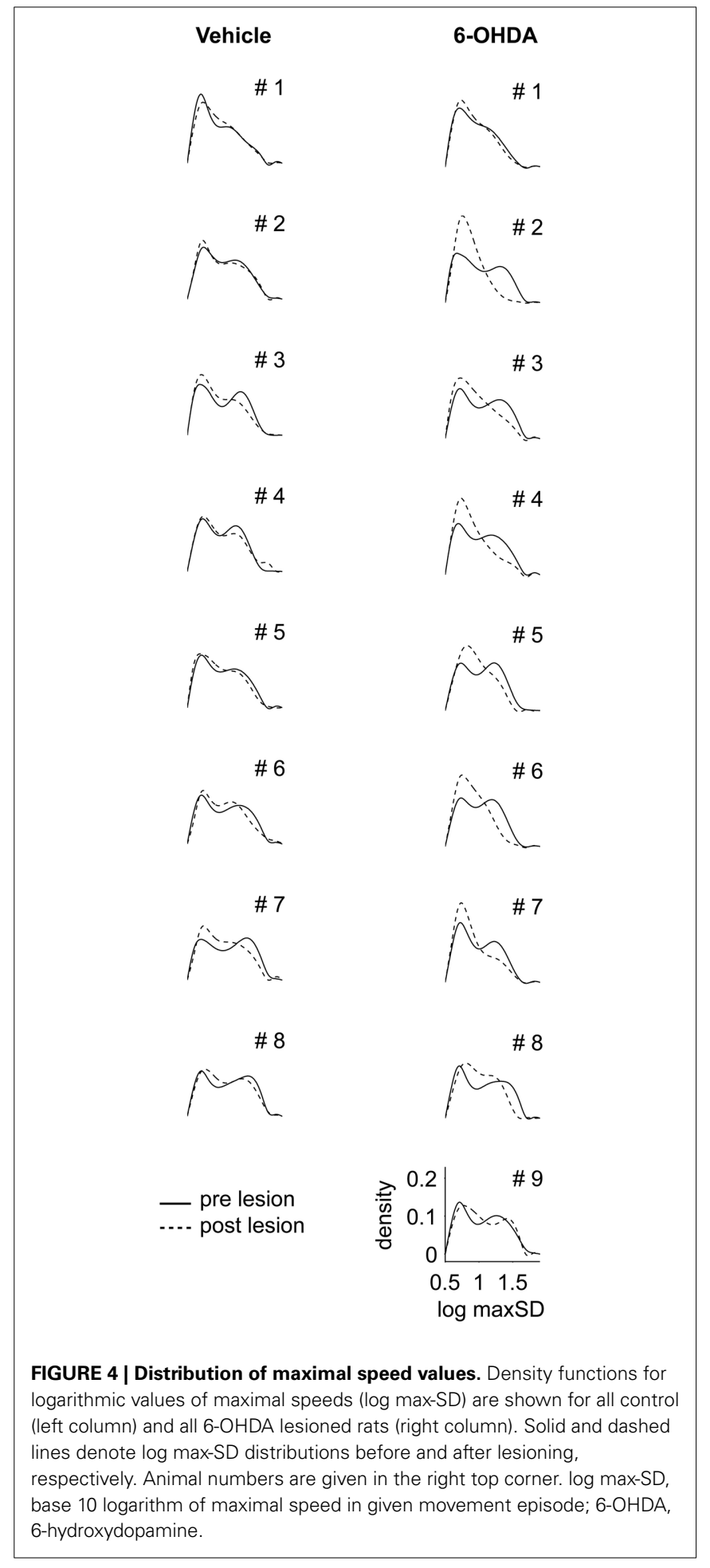

was significant also within the PD group at 12 days of recovery $(P=0.02$ at 12 days vs. $P=0.18$ at 28 days; $P=0.92$ for 12 vs. 28 days; corrected). We found no significant difference for first gear episodes $(P=0.07$ pre- vs. $12+28$ days postoperatively, $P=0.25$ at 12 days, $P=0.09$ at 28 days; $P=0.26$ for 12 vs. 28 days; corrected). 

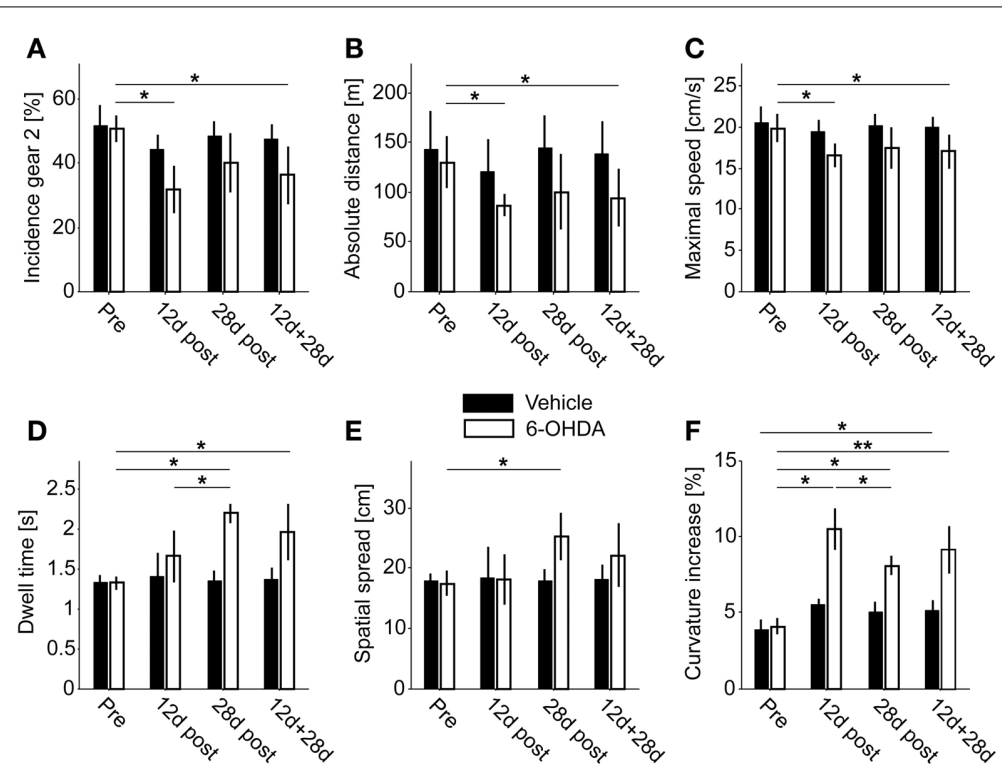

FIGURE 5 | Behavioral endpoints. (A) Incidence of second gear episodes. (B) Absolute covered distance. (C) Average maximal movement speed of second gear episodes. (D) Dwell time of second gear episodes. (E) Spatial spread of second gear episodes. (F) Increase of curvature in second gear episodes. Single asterisks denote $P \leq 0.05$, double asterisks denote $P \leq 0.001$ (corrected).

\section{DWELL TIME}

The average dwell time of second gear episodes was increased in lesioned animals but not in controls (PD rats: $1.32 \pm 0.08 \mathrm{~s}$ vs. $1.96 \pm 0.35 \mathrm{~s}$, pre- vs. $12+28$ days postoperatively, $P=0.01$; control rats: $1.33 \pm 0.09$ s vs. $1.37 \pm 0.15$ s, pre- vs. $12+28$ days postoperatively, $P=0.84$, corrected; Figure 5D). Interestingly, the difference in dwell time was also significant in the 28 days subset of PD rats, but not at 12 days post-surgery $(P=0.17$ at 12 days vs. $P=0.01$ at 28 days; $P=0.05$ for 12 vs. 28 days; corrected). Furthermore, the dwell time of first gear episodes was also significantly prolonged $(P=0.001$ pre- vs. $12+28$ days postoperatively, $P=0.01$ at 12 days, $P=0.008$ at 28 days; $P=0.98$ for 12 vs. 28 days; corrected).

\section{SPATIAL SPREAD}

The spatial spread accomplished within a given episode (averaged across all second gear episodes of full motion) was increased after 6-OHDA lesioning (PD rats: $17.5 \pm 2 \mathrm{~cm}$ vs. $22.1 \pm 5.3 \mathrm{~cm}$, pre- vs. $12+28$ days postoperatively, $P=0.08$; control rats: $18 \pm$ $1.2 \mathrm{~cm}$ vs. $18 \pm 2.6 \mathrm{~cm}$, pre- vs. postoperatively, $P=0.83$, corrected; Figure 5E). Comparable to the dwell time, the difference was significant at 28 days in PD-rats, but not at 12 days or within the combined group $(P=1$ at 12 days vs. $P=0.01$ at 28 days; $P=0.08$ for 12 vs. 28 days; corrected). Similar to the dwell time, the spatial spread was also significantly enlarged for first gear episodes $(P=0.001$ pre- vs. $12+28$ days postoperatively, $P=0.02$ at 12 days, $P=0.008$ at 28 days; $P=0.98$ for 12 vs. 28 days; corrected).

\section{CURVATURE}

6-OHDA injections led to an increase of the mean curvature of motion tracks (PD-rats: $4.07 \pm 0.52 \%$ vs. $9.15 \pm 1.66 \%$, pre- vs.
$12+28$ days postoperatively, $P=0.002)$. Notably, the curvature was also significantly increased in vehicle injected control rats, but only for the comparison of pre vs. combined postoperative groups $(3.850 \pm 0.70 \%$ vs. $5.15 \pm 0.64 \%$, pre- vs. $12+28$ days postoperatively, $P=0.02$, corrected; Figure 5F). However, in $\mathrm{PD}$ rats we saw a stronger increase 12 days after injections $(P=0.02$ at 12 days $)$ and a significant recovery of curvature ratios 2 weeks later $(P=0.05$ for 12 vs. 28 days). At the later time point the ratio was still significantly enhanced ( $P=0.01$ at 28 days). The same pattern of statistically significant differences was observed for first gear episodes $(P=0.001$ pre- vs. $12+28$ days postoperatively, $P=0.01$ at 12 days, $P=0.008$ at 28 days; $P=0.05$ for 12 vs. 28 days; corrected).

\section{BOOTSTRAP REGRESSIONS}

We found a significant correlation for five of 55 independent comparisons (Figure 6A) in 6-OHDA treated rats $(n=8)$. The estimated number of VTA, but not of $\mathrm{SNc}$ neurons correlated negatively with the magnitude of weight reduction $(R=-0.86$, Figure 6B1). Weight loss also correlated with dwell time $(R=0.8$, Figure 6B2) and spatial spread $(R=0.8$, Figure 6B3). Spatial spread also correlated with the incidence of second gear episodes ( $R=0.71$, Figure 6B4). Furthermore, the curvature of motion tracks obtained postoperatively correlated negatively with the spatial spread $(R=-0.9$, Figure 6B5). Further 9 comparisons were found statistically significant before FDR-correction and yielded $P$-values $<0.1$ after correction (Figure 6A). Among them was the only comparison, incidence of second gear episodes vs. maximal speed, that was also found highly correlated in vehicle injected controls $(R=1$, data not shown). 

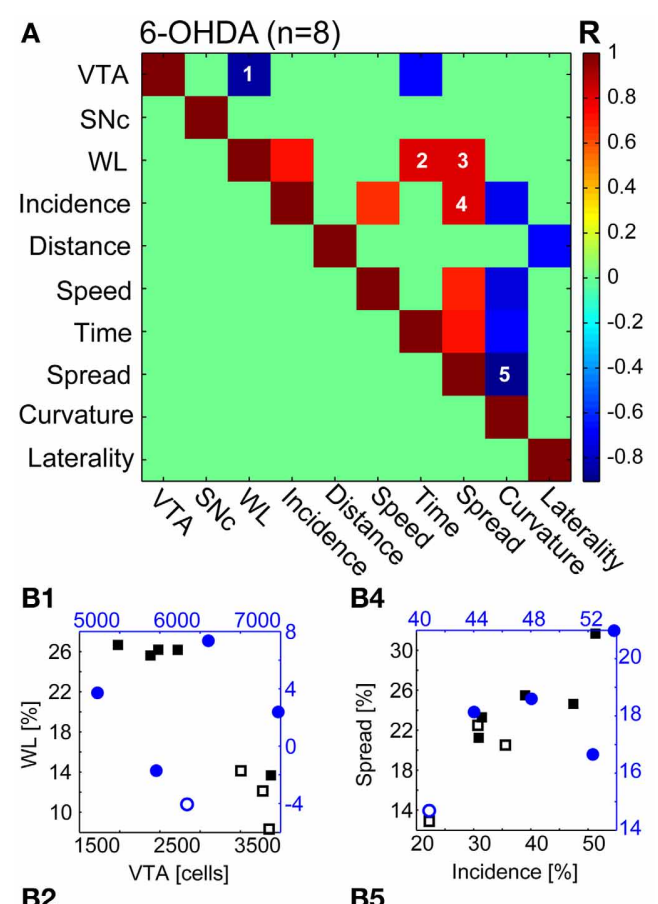

B2
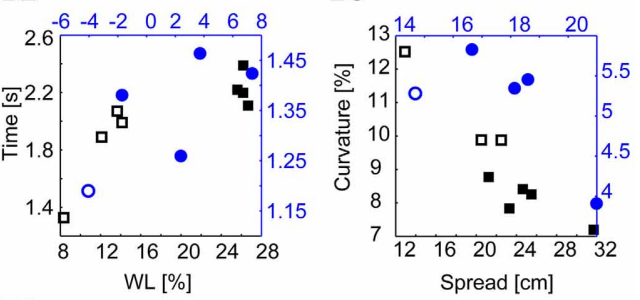

B3

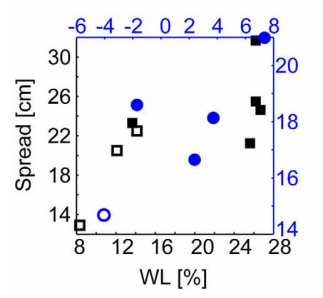

口 6-OHDA (12d)

- 6-OHDA (28d)

- Vehicle (12d)

- Vehicle (28d)

FIGURE 6 | Bootstrap regressions. (A) Independent bootstrap regressions ( $n=55$ ) of the 6-OHDA group ( $n=8$ animals). The bootstrapping $R$-value is color-coded and clipped for correlations with $P>0.1$ after FDR-correction (green fields). (B1-5) Correlations with $P<0.05$ after FDR-correction. Open and filled markers indicate rats from 12 to 28 days subsets, respectively. 6-OHDA, 6-hydroxydopamine.

\section{DISCUSSION}

The main findings of our study are that the stereotypical locomotion pattern of rats exhibiting a first and a second gear of motion is partially lost after bilateral dopaminergic denervation. The reduced incidence of second gear episodes points at distinct deficits in the execution of fast motor sequences. However, although generally slowed, bilaterally lesioned rats also displayed bouts of spontaneous locomotion, to some degree similar to kinesia paradoxa observed in PD patients. Contrary to expectation, we observed an abnormally increased spatial spread and increased

motion time during episodes of full motion in lesioned rats. Moreover, we noted larger curvature values in movement paths of lesioned rats. Upon acceleration, curvature values showed a reduction toward physiological values. All these changes were accompanied by an alteration of circadian locomotor activity.

The depletion of estimated dopaminergic cell populations in the SNc and VTA in our disease model was comparable to that observed in humans suffering from advanced PD, where the VTA is known to be significantly less depleted than the SNc (Hirsch et al., 1988). However, the mesocorticolimbic dopamine projections are vulnerable in advanced PD as well, and seem to contribute to the complex clinical picture encountered in humans, especially at later disease stages (Thobois et al., 2010). It is worth mentioning that our stereological results were similar to cell-loss estimates of $40-50 \%$ in the VTA described in human postmortem studies (Uhl et al., 1985; Hirsch et al., 1988; Dymecki et al., 1996; McRitchie et al., 1997; Thobois et al., 2010). The estimated number of SNc neurons of vehicle injected rats in our study was comparable to the number of TH-positive cells reported by a previous study (Fox et al., 2001) that investigated Fisher $344 \times$ Brown Norway hybrids. Other studies reported significantly higher THpositive cell counts in different rat strains (Lewis rats, Strackx et al., 2008; Long-Evans rats Healy-Stoffel et al., 2012; SpragueDawley rats, Walker et al., 2012) suggesting large differences in the absolute number of midbrain dopaminergic cells in different rat strains.

PD rats in our study displayed a considerable reduction of body weight $(\sim 20 \%)$. Weight loss and associated metabolic changes might by themselves have an influence on locomotion. We included the reduction of body weight into multiple bootstrap regressions and investigated the interdependence between behavioral endpoints, dopaminergic cell loss and weight reduction. We found a significant negative correlation between the VTA, but not the SNc dopaminergic population, and the magnitude of weight loss. Thus, abnormal feeding behavior and more generally abulia, was related to cell loss within the mesolimbic dopaminergic system in our study (Redgrave et al., 2010).

Our analysis approach allowed the discrimination of naturally occurring motion episodes in severely dopamine depleted and overtly akinetic and bradykinetic rats. The characterization of motion episodes by the maximal rather than the average speed accomplished in a given episode enabled us to analyze rare and, given the pronounced parkinsonian phenotype of our rats, unexpected episodes of qualitatively altered motor activity. These motion episodes were still characterized by a reduced incidence (i.e., poverty of movement or akinesia), reduced overall traveled distance (i.e., hypokinesia) and reduced maximal speed (i.e., bradykinesia) compared to controls. Hence, important hallmark symptoms of PD were clearly detectable in motion episodes of 6OHDA rats. However, we did not see a significant correlation of these three behavioral endpoints with the estimated populations of the SNc and VTA.

Overall, the quantitative change in traveled distance was rather small. A disruption of the circadian rhythm, a cardinal non-motor symptom in human PD patients (Videnovic and Golombek, 2013) and present in animal models of PD (Kudo et al., 2011; Willison et al., 2013) may introduce a bias. Higher 
absolute activity values may result from prolongation and fragmentation of activity and reduced sleep phases. Our analysis method could not differentiate between awake resting and true sleep, but conclusions could still be drawn from the overall circadian activity pattern. Here, we observed a more fragmented pattern of activity together with a nearly absent second activity phase. Moreover, PD rats showed a significantly reduced absolute number of motion episodes in comparison to controls (data not shown). Together, this argues against a circadian bias toward higher distance values in our data.

The finding of increased dwell times and spatial spread in first and second gear motion episodes of PD rats was rather unexpected. The spatial spread in the 6-OHDA group was increased to values not seen in any pre-lesion or control recordings. Theoretically, an increased dwell time could be explained as a function of slowed locomotion. To reach the same location, bradykinetic rats may simply need more time. This interdependence should theoretically manifest in a negative correlation between speed and dwell time or spatial spread. To the contrary, our data revealed a positive correlation between speed and spatial spread, albeit not significant after FDR-correction. That is, faster PD rats showed spatially extended second gear episodes. Could a postlesion increase in curvature explain increased dwell times? Arguing against that, curvature values were significantly negatively correlated with spatial spread, and also with dwell time, speed and incidence of second gear episodes (before FDRcorrection). Furthermore, a higher incidence of second gear behavior correlated significantly with prolonged spatial spread. Such an increased incidence could be the result of an uneven effect of 6-OHDA lesioning and stronger diminishment of first gear in comparison to second gear activity. This would also be supported by the finding that 6-OHDA induced weight loss correlated positively with the incidence of second gear activity (before FDR-correction). Taken together, increased spatial spread and dwell times constitute an abnormal behavioral characteristic of PD rats.

An abnormal increase of locomotor activity in bilaterally lesioned rats has been described in response to a pharmacological challenge (Schallert et al., 1978). Unexpected bursts of locomotion in otherwise akinetic patients are also well known to occur in selected PD patients or cases with postencephalitic parkinsonism (kinesia paradoxa). The sudden ability of our rats to cover longer distances within a motion episode may thus represent a rat equivalent of this condition. There are, however, important differences to classical kinesia paradoxa, which is often triggered by external sensory cues (Martin, 1967).

One possible explanation for prolonged dwell times and increased spatial spread could be problems with the termination of movement. In PD patients, deficiency in smooth motion termination is known (Dounskaia et al., 2009), along with an inability to promptly change generated force or quickly re-plan current motion. Typically, PD patients show an asymmetric evolution of velocities during the execution of goal-directed behaviors with initial fast accelerations (Flash et al., 1992). In our data we found a symmetric evolution of acceleration between PD and control rats. That is, we saw a linear relationship between the dwell time and the time point where PD or control rats accomplished their maximal speed in a given episode (data not shown). Thus, the observed behavioral abnormalities do not support the presence of the hastening phenomenon (unwanted acceleration of movement in human PD patients) in our rats. Finally, it is also conceivable that the abnormal drive to continue exploration or food search could be a behavioral consequence of metabolic changes accompanying weight loss (Redgrave et al., 2010).

Another abnormal feature was found in the movement path's curvature. The ratio between real and direct distance of first and second gear motion episodes was significantly enlarged in $\mathrm{PD}$ rats and, to a lesser extent, in controls. What could be the cause of increased curvatures? Unilaterally depleted rats display a spontaneous ipsiversive motor bias (Ungerstedt, 1971). Hence, curvature increases could result from spontaneous partial turning behavior provoked by asymmetric dopaminergic lesioning in our rats. However, the calculated LI did not correlate with curvature or any other behavioral endpoint except distance (before FDR-correction). Furthermore, unilateral 6-OHDA lesions were shown to shorten steps in spontaneous walking (Metz et al., 2005), thus modeling the shuffling gait of PD patients (Knutsson, 1972). Hind limb rigidity was detected upon manual assessment in our PD rats and could have contributed to disturbed locomotor patterns with reduced step sizes, axial instability and loss of balance during walking. Hemiparkinsonian rats, when tested by e.g., beam-walking, also exhibit difficulties in motor coordination (Truong et al., 2006). Interestingly, we found a significant negative correlation between curvature and spatial spread, as well as maximal speed, dwell time and incidence of second gear activity (before FDR-correction). Thus, faster running or greater spreads were associated with straighter movement paths in PD rats. If limb rigidity was indeed related to the expression of increased curvature values, then the ability of PD rats to generate fast motion with reduced curvature values may reflect an overcoming of rigidity for brief periods.

We saw some differences between lesioned animals that were monitored at an early (12 days) and later stage (28 days) after 6-OHDA lesioning. Most strikingly, the dwell time was significantly longer at 28 days in comparison with 12 days postlesion. Contrary, curvature increases significantly decreased again at the later point of investigation. Importantly, no significant difference in cell counts was observed between the two subsets. It remains difficult to infer which compensatory mechanisms were at work here. The milder but still significant increase of curvature in controls, in conjunction with the significant decrease of curvature values in PD rats with time, could argue for an influence of and recovery from surgery per se. The emergence of an abnormally increased behavior such as kinesia paradoxa may have in turn developed over time when the rats recovered fully from surgery. Despite putative effects of recovery from surgery, absolute distance, speed and incidence of second gear activity were comparably decreased at an early and later point of investigation.

We conclude that long-term behavioral observations of spontaneous locomotion offers new perspectives on distinctly different modes of motion in a rat model of advanced PD. The present behavioral analysis, in conjunction with in-vivo electrophysiology, may be particularly suited to reveal neural mechanisms underlying motor fluctuations such as kinesia 
paradoxa, and may provide further insights into the complex pathophysiology of PD.

\section{AUTHOR CONTRIBUTIONS}

Benjamin Grieb, Gerhard Engler, Ismini Papageorgiou, Wolfgang Hamel, and Christian K. Moll designed research; Benjamin Grieb, Gerhard Engler, and Ismini Papageorgiou performed experiments; Benjamin Grieb, Constantin von Nicolai, Andrew Sharott, and Ismini Papageorgiou analyzed the data; Benjamin Grieb, Constantin von Nicolai, Andrew Sharott, Andreas K. Engel, and Christian K. Moll wrote the manuscript.

\section{ACKNOWLEDGMENTS}

The authors would like to thank Doris Lange for help with the histology. This work was supported by the European Union (MRTN-CT-2005-019247).

\section{REFERENCES}

Avila, I., Parr-Brownlie, L. C., Brazhnik, E., Castañeda, E., Bergstrom, D. A., and Walters, J. R. (2010). Beta frequency synchronization in basal ganglia output during rest and walk in a hemiparkinsonian rat. Exp. Neurol. 221, 307-319. doi: 10.1016/j.expneurol.2009.11.016

Belujon, P., Bezard, E., Taupignon, A., Bioulac, B., and Benazzouz, A. (2007). Noradrenergic modulation of subthalamic nucleus activity: behavioral and electrophysiological evidence in intact and 6-hydroxydopamine-lesioned rats. J. Neurosci. 27, 9595-9606. doi: 10.1523/JNEUROSCI.2583-07.2007

Benjamini, Y., and Hochberg, Y. (1995). Controlling the false discovery rate: a practical and powerful approach to multiple testing. J. R Stat. Soc. B 57, 289-300. doi: $10.2307 / 2346101$

Bergman, H., Wichmann, T., and DeLong, M. R. (1990). Reversal of experimental parkinsonism by lesions of the subthalamic nucleus. Science 249, 1436-1438. doi: 10.1126/science. 2402638

Brazhnik, E., Cruz, A. V., Avila, I., Wahba, M. I., Novikov, N., Ilieva, N. M., et al. (2012). State-dependent spike and local field synchronization between motor cortex and substantia nigra in hemiparkinsonian rats. J. Neurosci. 32, 7869-7880. doi: 10.1523/JNEUROSCI.0943-12.2012

Cass, W. A., Peters, L. E., and Smith, M. P. (2005). Reductions in spontaneous locomotor activity in aged male, but not female, rats in a model of early Parkinson's disease. Brain Res. 1034, 153-161. doi: 10.1016/j.brainres.2004.12.009

Cenci, M A., Whishaw, I. Q., and Schallert, T. (2002). Animal models of neurological deficits: how relevant is the rat? Nat. Rev. Neurosci. 3, 574-579. doi: 10.1038/nrn877

Dounskaia, N., Fradet, L., Lee, G., Leis, B. C., and Adler, C. H. (2009). Submovements during pointing movements in Parkinson's disease. Exp. Brain Res. 193, 529-544. doi: 10.1007/s00221-008-1656-6

Drai, D., Benjamini, Y., and Golani, I. (2000). Statistical discrimination of natural modes of motion in rat exploratory behavior. J. Neurosci. Methods 96, 119-131. doi: 10.1016/S0165-0270(99)00194-6

Drai, D., and Golani, I. (2001). SEE: a tool for the visualization and analysis of rodent exploratory behavior. Neurosci. Biobehav. Rev. 25, 409-426. doi: 10.1016/S0149-7634(01)00022-7

Drai, D., Kafkafi, N., Benjamini, Y., Elmer, G., and Golani, I. (2001). Rats and mice share common ethologically relevant parameters of exploratory behavior. Behav. Brain Res. 125, 133-140. doi: 10.1016/S0166-4328(01)00290-X

Dymecki, J., Lechowicz, W., Bertrand, E., and Szpak, G. M. (1996). Changes in dopaminergic neurons of the mesocorticolimbic system in Parkinson's disease. Folia Neuropathol. 34, 102-106.

Ferro, M. M., Bellissimo, M. I., Anselmo-Franci, J. A., Angellucci, M. E., Canteras, N. S., and Da Cunha, C. (2005). Comparison of bilaterally 6-OHDA- and MPTP-lesioned rats as models of the early phase of Parkinson's disease: histological, neurochemical, motor and memory alterations. J. Neurosci. Methods 148, 78-87. doi: 10.1016/j.jneumeth.2005.04.005

Flash, T., Inzelberg, R., Schechtman, E., and Korczyn, A. D. (1992). Kinematic analysis of upper limb trajectories in Parkinson's disease. Exp. Neurol. 118, 215-226. doi: 10.1016/0014-4886(92)90038-R
Fox, C. M., Gash, D. M., Smoot, M. K., and Cass, W. A. (2001). Neuroprotective effects of GDNF against 6-OHDA in young and aged rats. Brain Res. 896, 56-63. doi: 10.1016/S0006-8993(00)03270-4

Gundersen, H. J. (1986). Stereology of arbitrary particles. a review of unbiased number and size estimators and the presentation of some new ones, in memory of William R. Thompson. J. Microsc. 143(Pt 1), 3-45. doi: 10.1111/j.13652818.1986.tb02764.x

Gundersen, H. J., and Jensen, E. B. (1987). The efficiency of systematic sampling in stereology and its prediction. J. Microsc. 147(Pt 3), 229-263. doi: 10.1111/j.1365-2818.1987.tb02837.x

Healy-Stoffel, M., Ahmad, S. O., Stanford, J. A., and Levant, B. (2012). A novel use of combined tyrosine hydroxylase and silver nucleolar staining to determine the effects of a unilateral intrastriatal 6-hydroxydopamine lesion in the substantia nigra: a stereological study. J. Neurosci. Methods 210, 187-194. doi: 10.1016/j.jneumeth.2012.07.013

Hirsch, E., Graybiel, A. M., and Agid, Y. A. (1988). Melanized dopaminergic neurons are differentially susceptible to degeneration in Parkinson's disease. Nature 334, 345-348. doi: 10.1038/334345a0

Kelly, P. H. (1975). Unilateral 6-hydroxydopamine lesions of nigrostriatal or mesolimbic dopamine-containing terminals and the drug-induced rotation of rats. Brain Res. 100, 163-169. doi: 10.1016/0006-8993(75)90253-X

Knutsson, E. (1972). An analysis of Parkinsonian gait. Brain 95, 475-486. doi: 10.1093/brain/95.3.475

Kudo, T., Loh, D. H., Truong, D., Wu, Y., and Colwell, C. S. (2011). Circadian dysfunction in a mouse model of Parkinson's disease. Exp. Neurol. 232, 66-75. doi: 10.1016/j.expneurol.2011.08.003

Martin, J. P. (1967). The Basal Ganglia and Posture. Philadelphia, PA: Lippincott.

McRitchie, D. A., Cartwright, H. R., and Halliday, G. M. (1997). Specific A10 dopaminergic nuclei in the midbrain degenerate in Parkinson's disease. Exp. Neurol. 144, 202-213. doi: 10.1006/exnr.1997.6418

Metz, G. A., Tse, A., Ballermann, M., Smith, L. K., and Fouad, K. (2005). The unilateral 6-OHDA rat model of Parkinson's disease revisited: an electromyographic and behavioural analysis. Eur. J. Neurosci. 22, 735-744. doi: 10.1111/j.14609568.2005.04238.x

Mokrý, J. (1995). Experimental models and behavioural tests used in the study of Parkinson's disease. Physiol. Res. 44, 143-150.

Paxinos, G., and Watson, C. (2005). The Rat Brain in Stereotaxic Coordinates. 5 th Edn. Sidney, NSW: Academic Press.

Redgrave, P., Rodriguez, M., Smith, Y., Rodriguez-Oroz, M. C., Lehericy, S., Bergman, H., et al. (2010). Goal-directed and habitual control in the basal ganglia: implications for Parkinson's disease. Nat. Rev. Neurosci. 11, 760-772. doi: $10.1038 / \mathrm{nrn} 2915$

Sakai, K., and Gash, D. M. (1994). Effect of bilateral 6-OHDA lesions of the substantia nigra on locomotor activity in the rat. Brain Res. 633, 144-50. doi: 10.1016/0006-8993(94)91533-4

Schallert, T., Whishaw, I. Q., Ramirez, V. D., and Teitelbaum, P. (1978). Compulsive, abnormal walking caused by anticholinergics in akinetic, 6-hydroxydopaminetreated rats. Science 199, 1461-1463. doi: 10.1126/science.564552

Schwarting, R. K., and Huston, J. P. (1996a). Unilateral 6-hydroxydopamine lesions of meso-striatal dopamine neurons and their physiological sequelae. Prog. Neurobiol. 49, 215-266. doi: 10.1016/S0301-0082(96)00015-9

Schwarting, R. K., and Huston, J. P.(1996b). The Unilateral 6-hydroxydopamine lesion model in behavioral brain research. Analysis of functional deficits, recovery and treatments. Prog. Neurobiol. 50, 275-331. doi: 10.1016/S03010082(96)00040-8

Seghier, M. L. (2008). Laterality index in functional MRI: methodological issues. Magn. Reson. Imaging 26, 594-601. doi: 10.1016/j.mri.2007.10.010

Strackx, E., Van den Hove, D. L., Steinbusch, H. P., Steinbusch, H. W., Vles, J. S., Blanco, C. E., et al. (2008). A combined behavioral and morphological study on the effects of fetal asphyxia on the nigrostriatal dopaminergic system in adult rats. Exp. Neurol. 211, 413-422. doi: 10.1016/j.expneurol.2008.02.006

Thobois, S., Ardouin, C., Lhommée, E., Klinger, H., Lagrange, C., Xie, J., et al. (2010). Non-motor dopamine withdrawal syndrome after surgery for Parkinson's disease: predictors and underlying mesolimbic denervation. Brain 133(Pt. 4), 1111-1127. doi: 10.1093/brain/awq032

Truong, L., Allbutt, H., Kassiou, M., and Henderson, J. M. (2006). Developing a preclinical model of Parkinson's disease: a study of behaviour in rats with graded 6-OHDA lesions. Behav. Brain Res. 169, 1-9. doi: 10.1016/j.bbr.2005. 11.026 
Uhl, G. R., Hedreen, J. C., and Price, D. L. (1985). Parkinson's disease: loss of neurons from the ventral tegmental area contralateral to therapeutic surgical lesions. Neurology 35, 1215-1218.

Ungerstedt, U. (1971). Postsynaptic supersensitivity after 6-hydroxy-dopamine induced degeneration of the nigro-striatal dopamine system. Acta Physiol. Scand. Suppl. 367, 69-93.

Videnovic, A., and Golombek, D. (2013). Circadian and sleep disorders in Parkinson's disease. Exp. Neurol. 243, 45-56. doi: 10.1016/j.expneurol.2012.08.018

Walker, Q. D., Johnson, M. L., Van Swearingen, A. E., Arrant, A. E., Caster, J. M., and Kuhn, C. M. (2012). Individual differences in psychostimulant responses of female rats are associated with ovarian hormones and dopamine neuroanatomy. Neuropharmacology 62, 2267-2277. doi: 10.1016/j.neuropharm.2012.01.029

West, M. J. (2002). Design-based stereological methods for counting neurons. Prog. Brain Res. 135, 43-51. doi: 10.1016/S0079-6123(02)35006-4

West, M. J., Slomianka, L., and Gundersen, H. J. (1991). Unbiased stereological estimation of the total number of neurons in thesubdivisions of the rat hippocampus using the optical fractionator. Anat. Rec. 231, 482-497. doi: 10.1002/ar.1092310411

Willison, L. D., Kudo, T., Loh, D. H., Kuljis, D., and Colwell, C. S. (2013). Circadian dysfunction may be a key component of the non-motor symptoms of
Parkinson's disease: insights from a transgenic mouse model. Exp. Neurol. 243, 57-66. doi: 10.1016/j.expneurol.2013.01.014

Conflict of Interest Statement: The authors declare that the research was conducted in the absence of any commercial or financial relationships that could be construed as a potential conflict of interest.

Received: 01 September 2013; accepted: 08 November 2013; published online: 27 November 2013.

Citation: Grieb B, von Nicolai C, Engler G, Sharott A, Papageorgiou I, Hamel W, Engel AK and Moll CK (2013) Decomposition of abnormal free locomotor behavior in a rat model of Parkinson's disease. Front. Syst. Neurosci. 7:95. doi: 10.3389/fnsys. 2013.00095

This article was submitted to the journal Frontiers in Systems Neuroscience. Copyright (C) 2013 Grieb, von Nicolai, Engler, Sharott, Papageorgiou, Hamel, Engel and Moll. This is an open-access article distributed under the terms of the Creative Commons Attribution License (CC BY). The use, distribution or reproduction in other forums is permitted, provided the original author(s) or licensor are credited and that the original publication in this journal is cited, in accordance with accepted academic practice. No use, distribution or reproduction is permitted which does not comply with these terms. 